devote their lives to searching for data to fit them.

Something should be said about the wider problems of conducting a congress of this kind successfully, that is, in such a manner as to ensure that lack of familiarity with the language of the speakers, poor acoustics and administrative blind spots do not add to the task of communicating a highly compressed technical report in a short interval of time. No facilities for simultaneous translation of the papers were available because of the prohibitive cost. Consequently, a number of participants--how many it is hard to say-failed to appreciate the papers in spite of possessing a good reading knowledge of the languages in which the papers were delivered. So much so that a proposal was submitted to the president by one momber that an investigation be undertaken of the intellectual effectiveness of the Congress. In these days when numerous international congresses are held in science, technology and medicine, the results of a study of this kind might well prove to be of widespread interest. Similar studies have been sponsored in the past by the World Health Organization and Unesco ${ }^{1}$.

Many of those who had attended international congresses of psychology on previous occasions doubtless felt that the scientific quality of the con. tributions and discussions marked a distinct advance. The eccentric fringe which used to be attracted to international assemblies of psychologists was happily small ; apart from one or two with highly characteristic opinions of their own, the mass of the members were indistinguishable from any other body of intellectual workers. All of them will recall with pleasure the reception at the Hôtel de Ville given by the Mayor of Brussels, and the paternal devotion lavished on them by Prof. Louis Delys, secretarygeneral of the Congress.

The Congress was preceded by a two-day international symposium on 'military psychology', held at the Palais des Académies and sponsored by the United States National Academy of Sciences and National Research Council at the request of the United States Air Force, Air Research and Development Command.

JoHN CoHeN

"The Technique of International Conferences". Internat. Soc. Sci. Bull., 5, 231 (1953).

\section{SOLVENT EFFECTS AND REACTION MECHANISM}

$\mathrm{F}^{\mathrm{s}}$

OR some years the Department of Chemistry of Queen Mary College, London, has organized symposia on the mechanisms of organic reactions. This year the specific topic of discussion was the effects of changes in medium on chemical reactions in solution. The moeting was held under the auspices of the Chemical Society on July 8 and 9. The title of the symposium was interpreted very broadly by both tho organizers and the speakers, and the subjects ranged from organic chemists' investigations of reaction products to a thermodynamic treatment of solutions. Sixteen papers were read; eight of them came from the United States, seven from British laboratories, and one from a Swiss laboratory.

The first session, under the chairmanship of Prof. E. D. Hughes, was largely concerned with the mechanisms of organic reactions. The first paper, by Prof. P. D. Bartlett of Harvard University, dealt with the relation between reaction-rates and solvent properties in terms of the short-range interactions between solvent molecules and the reactants and transition states. Prof. Bartlett brought out very clearly the inadequacy of any treatment which considers only the bulk dielectric constant of the solvent, but showed how the kinetic effects of graded changes in solvent properties, for example, acidity or deuterium content, could sometimes be used as mechanistic criteria. The hydrolysis of $\beta$-lactones was discussed in this context. Discussing this paper Prof. W. F. K. Wynne-Jones pointed out that the kinetic effects of changes in bulk dielectric constant will be of considerable importance for solvents of low dielectric constant. He also gave examples of the effects of solvent changes on acid-base equilibria.

The nature of solvolytic reactions the behaviour of which may be intermediate between $S_{N} 1$ and $S_{N} 2$ was discussed by Dr. V. Gold of King's College, London, with particular reference to the ways in which solvent molecules can interact with the reaction centre. The range of the subsequent discussion showed that there is no unanimity of opinion on this particular problem. It appears that the original definitions of Hughes and Ingold are sometimes used rather loosely, and their appropriate application limits considerably the territory in this 'no-man's land, between the two clear-cut mechanisms.

Dr. E. M. Kosower of the University of Wisconsin described the dependence of the charge-transfer spectrum of some alkyl-pyridinium iodides upon solvent composition. This dependence could be used for calculating an empirical mesture of solvent polarity, the $Z$ value, which could be correlated with the rates of some solvolytic reactions. Evidence on the nature of the carbonium ion intermediates in reactions and oxygen exchanges of some alcohols and glycols was given by Dr. C. A. Bunton of University College, London. The carbonium ions may be captured by water molecules, regenerating the original alcohol, and this is observed in the elimination of olefins, and in anionotropic rearrangements.

The kinetic effects which deuterium substitution on a $\beta$-carbon atom has on nucleophilic substitutions and eliminations were described by Dr. V. J. Shiner of the University of Indiana, and various explanations of these effects, involving inter alia solvent interactions with the polarized $\mathrm{C}-\mathrm{H}$ or $\mathrm{C}-\mathrm{D}$ bonds, were considered. Discussing this paper, Drs. Halevi and Lewis gave further evidence for the thermodynamic and kinetic effects of deuterium substitution.

The mechanisms of solvolyses of carboxylic acid amides and esters in sulphuric acid were then discussed by Dr. R. J. Gillespie of University College, London. These compounds are fully protonated in this highly acidic solvent, and unimolecular mechan. isms are faclitated, whereas in water and aquenusorganic solvents bimolecular substitutions are more common.

Prof. W. F. K. Wynne-Jones of King's College, Newcastle upon Tyne, was the chairman for the morning session of July 9. The first paper, by Dr. M. L. Bender of the Illinois Institute of Technology, was on the catalytic effect of Brönsted bases on the hydrolysis of esters and amides, and the evidence given showed that they acted as nucleophilic reagents by attacking the carbonyl carbon atom, creating entities which readily undergo further reaction. The catalytic power was much greater when these nucleophilic groups were already present 
in the molecule, and it was suggested that this catalysis might occur in some enzyme-catalysed hydrolyses.

The next three papers dealt with the intimate details of the interactions between solvent and solute molecules. Dr. P. A. M. Wyatt of the University of Sheffield discussed the possibility of accounting for the properties of a solution in terms of a specific or 'chemical' solvation. $\mathrm{He}$ quoted as examples the thermal properties of solutions in sulphuric and nitric acids, and pointed out that, although solvation is important, other factors have to be considered, and that their nature is not well understood. Dr. J. W. Smith of Bedford College, London, showed how measurements of dielectric constant can give evidence for hydrogen-bonding in liquid mixtures, and how steric hindrance in aromatic amines could affect their interaction with solvent molecules. A semiempirical treatment for the dependence of the rates of nucleophilic substitutions upon solvent properties was outlined by Dr. R. F. Hudson of Queen Mary College, London, who applied this treatment to the kinetics of alcoholyses of acyl halides in solvents of low polarity.

Spectrophotometric methods for investigation of carbonium ions in non-aqueous solvents were described by Prof. A. G. Evans of University College, Cardiff. His group has studied the formation of these ions from triaryl compounds, and it seems that there are specific interactions between the phenol molecules of the solvent and the carbonium ions. Similar measurements have been made on the carbonium ions formed by protonation of an olefin, or by the reaction between an alkyl halide and a metal halide. During discussion of this work Dr. R. H. Plesch suggested that these spectrophotometric measurements could be used to calculate approximate values for the solvation energies of carbonium ions.

The chair was taken by Prof. M. S. Newman of the University of Ohio during the afternoon session. An electrostatic theory for the kinetic effects of solvents was presented by Prof. E. S. Amis of the University of Arkansas, who suggested that the rates of many reactions could be correlated simply with the bulk dielectric constant of the solvents. Subsequent speakers showed that this simple electrostatic theory could not account for many of the complex solvent effects on rates and equilibria.

Dr. H. Zollinger read a paper by himself and Dr. O. A. Stamm on the kinetics of diazo-coupling reactions studied in the University of Basle. They found that kinetic salt and solvent effects could only be considered in terms of specific interactions between ions or solvent molecules and the groups present in the reactants. The authors were able to interpret the effects of Brönsted bases on the rates and chemical course of these reactions by considering their mechan. istic details.

The first of two papers on solvent effects in nonpolar reactions was given by Dr. J. E. Leffler of Florida State University, who outlined the form of the interactions between ions or solvent molecules and the reactants and transition states, and described a very detailed kinetic study of the rates of racemizations of a number of optically active diphenyls. Dr. G. A. Russell of the General Electric Corporation gave results on the photochemical chlorination of aliphatic hydrocarbons in conditions such that the first step of the reaction is the abstraction of a hydro. gen atom from the hydrocarbon. It was possible to use the variation of product composition with changes in the solvent as evidence for the formation of a complex between an electrophilic chlorine atom and an aromatic hydrocarbon. In discussing this work Dr. W. A. Waters showed that while some free atoms and radicals had electrophilic properties others, such as sodium atoms, could act as nucleophilic reagents. The paper by Prof. L. F. Hatch and his co-workers described a re-investigation which they had made of the products of the addition of bromine to butadiene in various solvents.

The wide range of work discussed at this symposium shows the interest which chemists are taking in the effects of solvent composition upon rates and mechanisms. Understanding of the short-range forces which govern the interactions between solvent molecules and solutes during the collisions in which chemical reactions occur is now needed. It was therefore unfortunate that the recent work on nucleophilic substitutions in benzene, a solvent in which these interactions have been studied, was not one of the subjects under discussion.

C. A. Bunton

\section{BLOCK AND GRAFT COPOLYMERS}

CONTINUING earlier seminars on polymer science in the Chemistry Department of University College, London (cf. Nature, 178, 18 ; 1956), five lectures on block and graft copolymers were given on July 15.

Prof. M. Szware (Syracuse University, New York) described experiments which show that anionic polymerization of certain vinyl monomers, such as styrene, carried out under appropriate conditions, does not involve a termination step (cf. Szware and collaborators, J. Amer. Chem. Soc., 79, 2026 ; 1957 ; and references to earlier communications given there). Polymers, formed in this manner, are able to continue their growth, if a fresh portion of monomer is added, after the complete conversion of the original monomer. If a monomer, $B$, is added to a block of 'living' polymer $A \ldots A$ a block copoly. mer is formed. The original polymer $A \ldots A$ may possess one or two 'living' ends, and therefore a variety of block copolymers can be prepared by relatively simple experimental techniques. The interaction of 'living' polymers with proton donors, carbon dioxide and oxygen was shortly discussed.

Dr. C. H. Bamford (Courtaulds, Ltd.) described a method for the preparation of block copolymers. A monomer, $A$, carrying a teritiary base residue, is converted into a polymer, $A \ldots A-\underbrace{\mathrm{Me}}_{\mathrm{N}(\mathbf{E t})_{2}}-\mathrm{H}$, which is afterwards used as a macromolecular transfer agent in the polymerization of a second monomer, $B$. Chain transfer to the terminal group of the first polymer results in the formation of a block copolymer $A \ldots A-B . B$. In a second method for preparing block copolymers, use is made of the following chain initiator : 